\title{
Is Aphthous Oral Ulceration Biopsy Always Useless?
}

\section{Yohan N'Guyen $^{\mathrm{a}}$, Amélie Servettaz ${ }^{\mathrm{a}}$, Florent Ehrhard ${ }^{\mathrm{b}}$, Firouze Bani-Sadr ${ }^{\mathrm{a}}$, Michel Pluot $^{\mathrm{c}}$}

\footnotetext{
a Internal Medicine Department, Infective Diseases and Clinical Immunology, Robert Debré Hospital, Reims, France

${ }^{\mathrm{b}}$ Hepatology and Gastroenterology Department, Robert Debré Hospital, Reims, France

${ }^{c}$ Pathological Anatomy Laboratory, Robert Debré Hospital, Reims, France
}

\begin{abstract}
:
Objective: To report a case of Behçet's disease whose diagnosis was only confirmed thanks to an oral aphthous lesion biopsy.
\end{abstract}

Materials and methods: Conventional histopathological analysis of a biopsy of an aphthous oral lesion that had appeared two days previously.

Results: A small vein vasculitis with eosinophil and neutrophil granulocytes was evidenced.

Conclusion: The presence of a small vein vasculitis was here strongly in favour of Behçet's disease, whereas such a diagnosis was not confirmed according to the International Study Group's criteria.

Keywords: Behçet's disease, aphthous ulceration, biopsy

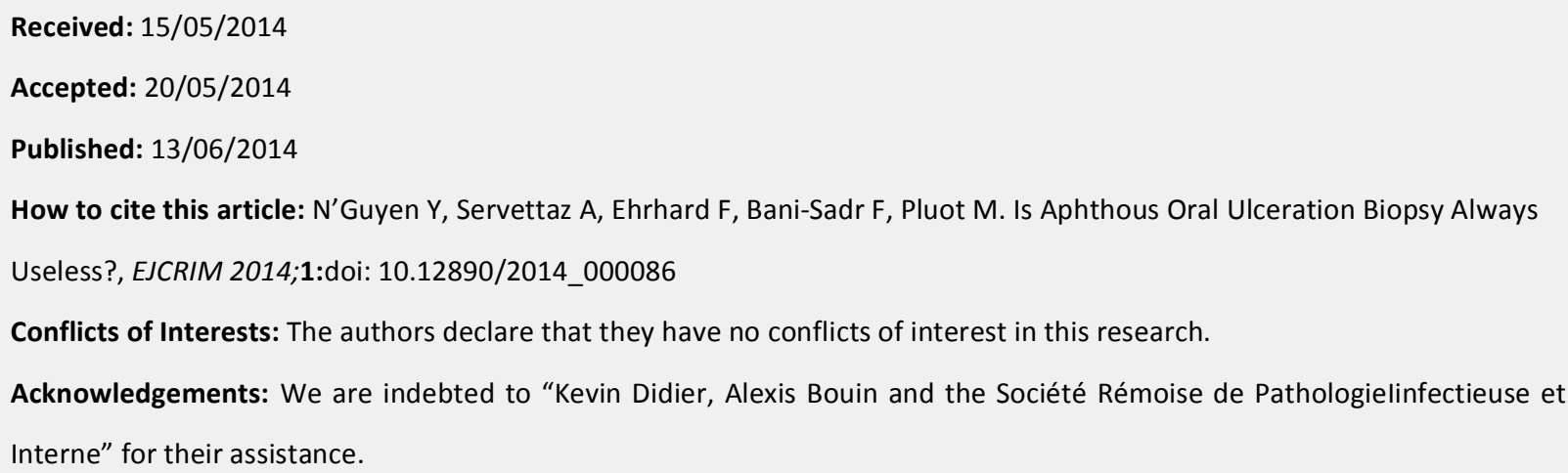


Aphthous oral ulceration is a frequent symptom that can be associated with a wide variety of pathologies, from benign to potentially life-threatening systemic diseases like Behçet's disease ${ }^{1}$. For this latter disease, the diagnosis is made upon converging clinical arguments ${ }^{1,2}$. We report the observation of a case of Behçet's disease whose diagnosis was confirmed thanks to histopathological analysis of the biopsy of an oral aphthous ulceration.

A 43-year-old man was referred to our institution because of asthenia associated with recurrent oral aphthous ulcerations. He took no medication and did not consume drugs, alcohol or tobacco. He worked as a personal care assistant for disabled people and did not report any recent exposure to pets or trips abroad. There was no significant medical history among his siblings and all four of his grandparents were of Western European origin (France).

His personal medical history was unremarkable (i.e., tonsillectomy) until the previous year, when he began to complain of chronic asthenia. This asthenia was intermittently worsening and was associated with arthralgia and back pain with morning stiffness, diarrhoea, feverish sensation, oral lesions and sometimes headache. Oral lesions were described as infracentimetric ovoid aphthous ulcerations that occurred sometimes on the dorsum of the tongue and usually resolved within 10-14 days (Fig. 1a).

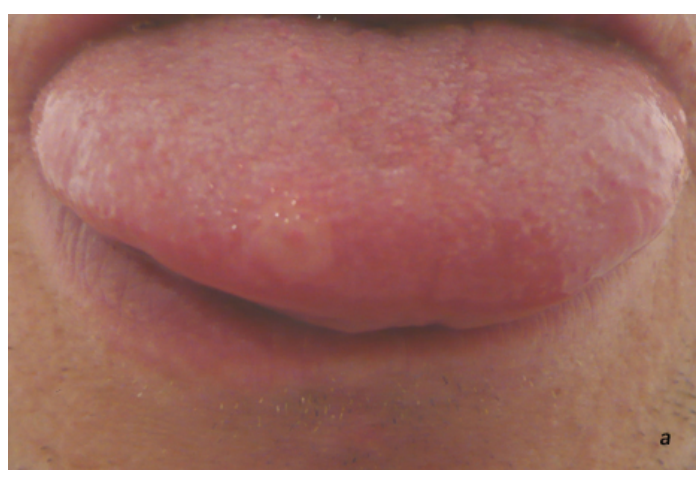

Figure 1a: Oral aphthous ovoid lesion of the dorsum of the tongue.
The patient had experienced three courses of these symptoms in the previous year and the time intervals between courses of the disease were characterized by a less severe long-lasting asthenia and minor oral ulcerations that resolved within 7 days.

Physical examination was unremarkable and no skin lesion was evidenced. The patient did not report aphthous genital lesions or uveitis. Inflammatory markers were slightly raised in blood (e.g., maximal C reactive protein $20 \mathrm{mg} / \mathrm{l}, \mathrm{N}<10 \mathrm{mg} / \mathrm{l}$ ).

Inflammatory bowel disease and enteropathic arthritis were first ruled out by faecal calprotectin assay, abdominal computed tomography scan, total colonoscopy, gastroscopy, capsule endoscopy and magnetic resonance imaging of the sacroiliac joint and the small bowel, which were all normal. Histological examinations of systematically performed gastric and colonic biopsies were normal. Lumbar puncture, brain magnetic resonance imaging and fundus examination were also normal. Pathergy test, blood cultures, human immunodeficiency virus serology and immunological assays including latex, Waaler-Rose and anti-nuclear antibodies were all negative. The patient did not show HLA B27 or HLA B51 haplotypes and plasma concentrations of folic acid, vitamins B12, C and D and 
immunoglobulins (classes and subclasses) were normal.

A biopsy of an aphthous lesion was performed 2 days after the onset of a new lesion on the labio-gingival sulcus and evidenced a small vein vasculitis with eosinophil and neutrophil granulocytes (Fig. 1b,c).

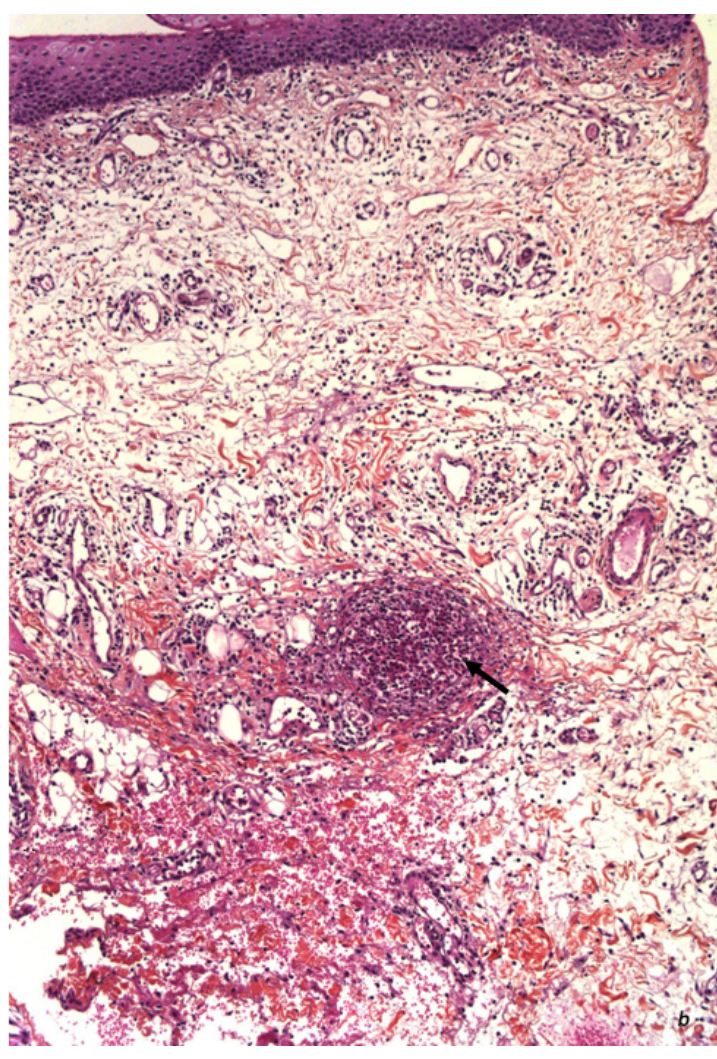

Figure $1 \mathrm{~b}$ : HE staining of the aphthous oral lesion biopsy; the black arrow shows small vein vasculitis with granulocytes $(\times 40)$.

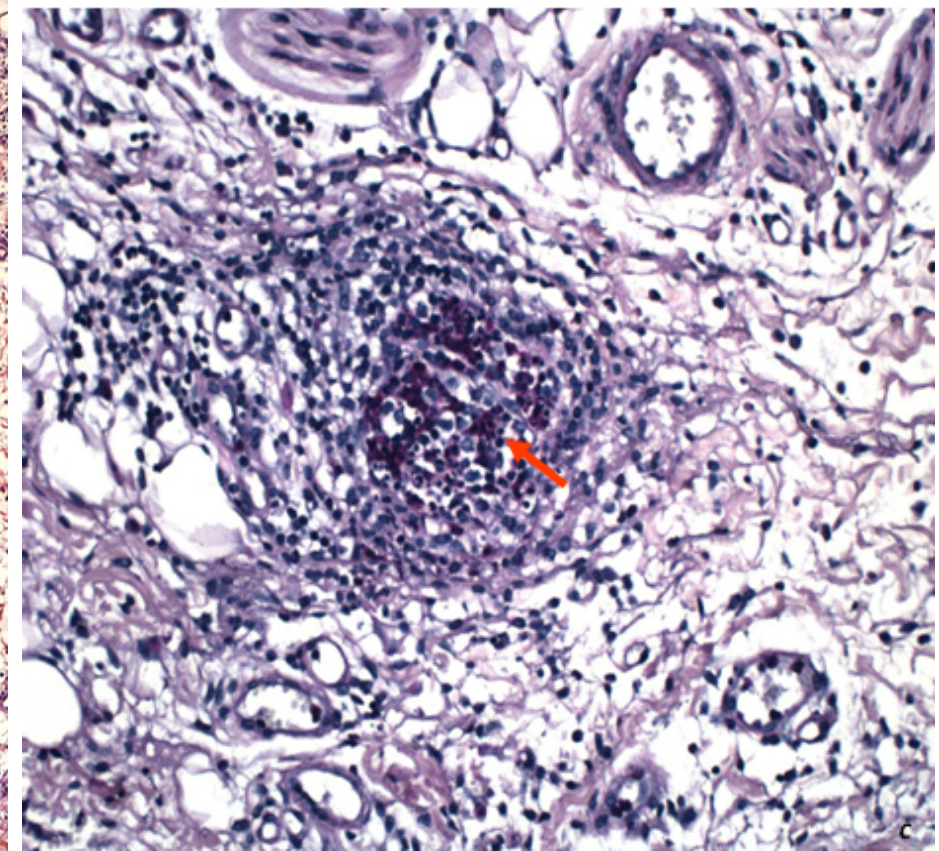

Figure 1c: PAS staining of the aphthous oral lesion biopsy; the red arrow shows small vein vasculitis with eosinophil granulocytes $(\times 100)$.

Taking these results together, Behçet's disease was diagnosed and a treatment combining colchicine and low-dose aspirin was prescribed. The outcome was favourable one year later, with an improvement of asthenia and a decrease in the number of courses of severe oral aphthous lesions. Herein, we report the observation of a case of Behçet's disease whose diagnosis was not established until an aphthous oral lesion biopsy was performed. The patient had no major criteria other than recurrent oral ulcerations and two minor suggestive criteria that were not sufficient for diagnosis according to the International Study Group classification ${ }^{2}$. If that biopsy was not performed, a mistaken diagnosis of idiopathic oral aphthous lesions associated with irritable bowel syndrome would probably have been made. 
The presence of a small vein vasculitis was here strongly in favour of Behçet's disease ${ }^{3}$, but evidence of venous vasculitis in histopathological analysis of oral aphthous lesions is not included in the classical diagnosis criteria of Behçet's disease ${ }^{2}$. Indeed, histopathology of skin or oral aphthous lesions is usually not relevant for the diagnosis of Behçet's disease, with aspecific results in a majority of cases ${ }^{4}$.

We hypothesize that histopathology was relevant here because of the short time interval between the onset of the oral lesion and the biopsy. The usefulness of systematic biopsies of recently appeared $(<48-72 \mathrm{~h})$ oral aphthous ulcerations in Behçet's disease and the predictive values of venous vasculitis in histopathological analysis of oral aphthous ulcerations for the diagnosis of Behçet's disease should be addressed in larger series of patients.

\section{Learning Points}

- The diagnosis of Behçet's disease is made upon converging clinical arguments according to the International Study Group criteria.

- Herein, we report the observation of a case of Behçet's disease, whose diagnosis was not established until an aphthous oral lesion biopsy was performed.

- The presence of a small vein vasculitis was strongly in favour of Behçet's disease in this case.

\section{References}

1. Scully C. Aphthous ulceration, N. Engl. J. Med. 2006;355:165-172.

2. International Study Group for Behçet's Disease. Criteria for diagnosis of Behcet's disease, Lancet 1990;335:1078-1080.

3. Chen KR, Kahawara Y, Miyakawa S, Nishikawa T. Cutaneous vasculitis in Behcet's disease: a clinical and histopathologic study of 20 patients, J. Am. Acad. Dermatol. 1997;36:689-696.

4. Melikoglu M, Kural-Seyahi E, Tascilar K, Yazici H. The unique features of vasculitis in Behçet's syndrome, Clin. Rev. Allergy Immunol. 2008;35:40-46. 